\title{
Irisin enhances chondrogenic differentiation of human mesenchymal stem cells via Rap1/PI3K/AKT axis.
}

\section{Taiqiu Chen}

Sun Yat-Sen Memorial Hospital

\section{Yan Peng}

Sun Yat-Sen Memorial Hospital

Wenjun Hu

Sun Yat-Sen Memorial Hospital

Huihong Shi

Sun Yat-Sen Memorial Hospital

\section{Pengfei Li}

Sun Yat-Sen Memorial Hospital

\section{Yichen Que}

Sun Yat-Sen Memorial Hospital

Jincheng Qiu

Sun Yat-Sen Memorial Hospital

\section{Xianjian Qiu}

Sun Yat-Sen Memorial Hospital

\section{Bo Gao}

Sun Yat-Sen Memorial Hospital

Hang Zhou

Sun Yat-Sen Memorial Hospital

\section{Yanbo Chen}

Sun Yat-Sen Memorial Hospital

\section{Yuanxin Zhu}

Sun Yat-Sen Memorial Hospital

\section{Shaoguang Li}

Sun Yat-Sen Memorial Hospital

\section{Anjing Liang}

Sun Yat-Sen Memorial Hospital

Wenjie Gao ( $\nabla$ gaowj7@mail.sysu.edu.cn )

Sun Yat-Sen Memorial Hospital https://orcid.org/0000-0001-9456-8884

\section{Dongsheng Huang}




\section{Research Article}

Keywords: irisin, human mesenchymal stem cells, chondrogenic differentiation, Rap1/PI3K/AKT signaling pathway, SIPA1L2, miR-125b-5p

Posted Date: January 31st, 2022

DOI: https://doi.org/10.21203/rs.3.rs-1285516/v1

License: (c) (i) This work is licensed under a Creative Commons Attribution 4.0 International License. Read Full License 


\section{Abstract}

\section{Background}

Human mesenchymal stem cells (hMSCs) have been proven to have inherent chondrogenic differentiation potential, which appears to be used in cartilage regeneration. Increasing evidence suggests that irisin enhances osteoblast differentiation of MSCs, but little is known about its potential on chondrogenic differentiation.

\section{Methods}

In our study, we investigated the effects of irisin on chondrogenic differentiation of hMSCs, and detected the underlying mechanism using RNA-seq.

\section{Results}

Compared with the controls, irisin treatment could significantly enhance chondrogenic differentiation of hMSCs. RNA-seq results prompted that irisin treatment activated the Rap1 and PI3K/AKT signaling pathway. Further studies demonstrated that irisin treatment could up-regulate the expression level of miR125b-5p, targeting SIPA1L2, consequently activating the Rap1/PI3K/AKT axis.

\section{Conclusions}

Collectively, our study reveals that irisin can enhance chondrogenic differentiation of hMSCs via the Rap1/PI3K/AKT pathway, suggesting that irisin possesses prospects in cartilage regeneration.

\section{Introduction}

Cartilage damage is very commonly encountered in patients with poor self-healing due to the lack of blood vessels, lymph, and nerves ${ }^{[1-2]}$. Many diseases including osteoarthritis, burns, and congenital deformities are characterized by cartilage defects, which lead to dysfunction and even disability ${ }^{[3-4]}$. Autologous cartilage transplantation has been a valid technique in repairing injured cartilage, which faces restricted proliferative capacity ${ }^{[5-6]}$. Thus, searching for suitable and effective methods to maintain the chondrocyte phenotype is essential for cartilage repair. Human mesenchymal stem cells (hMSCs) have been proven to be an effective cartilage tissue repair reagent due to their capacities of intrinsic regeneration and chondrogenic differentiation ${ }^{[7-8]}$.

Irisin is a polypeptide fragment formed by cleavage of type III fibronectin component including protein 5 (FNDC5) as the extracellular receptor ectodomain, which is widely secreted by multiple tissues such as skeletal muscle, liver, salivary glands, cardiomyocytes, and bone ${ }^{[9-11]}$. Accumulated studies have revealed that irisin was involved in many physiological processes, such as lipid and glucose homeostasis, anti-oxidation, and anti-tumor activities ${ }^{[12-15]}$. Regarding the regulation of development and metabolism of the skeletal system, irisin was discovered to possess a powerful regulative effect. In 
vivo, irisin treatment prevented loss of bone mass and improved bone metabolism ${ }^{[16-17]}$, while in vitro, irisin has been found to enhance the osteoblastogenetic process of MSCs, respectively ${ }^{[18-19]}$. In addition, $\mathrm{Li}$ et al. discovered that irisin was involved in the development of articular cartilage, and Wang et al. found that irisin displayed chondroprotective effects on the development of osteoarthritis ${ }^{[20-21]}$. Despite its considerable roles in the regulation of skeletal metabolism, it remains elusive whether irisin regulated the chondrogenic differentiation of hMSCs.

In our study, the ability to enhance chondrogenic differentiation of hMSCs of irisin and its potential underlying mechanism are evaluated. Herein, we report that irisin enhances chondrogenic differentiation of hMSCs by up-regulating the expression level of miR-125b-5p, targeting SIPA1L2, and activating Rap1/PI3K/AKT signaling. Our results provide abundant evidence for the use of irisin as a repair reagent on diseases with cartilage injury.

\section{Material And Methods}

\section{Isolation and culture of human mesenchymal stem cells}

As previously described, hMSCs were obtained from healthy volunteer donors, and isolated from bone marrow ${ }^{[22-24]}$. The bone marrow samples were diluted with PBS, and the cells were then fractionated. Then, the cells were resuspended in low-glucose DMEM, seeded, and incubated. The non-adherent cells were removed after $48 \mathrm{~h}$. When they reached approximately $80 \%$ confluence, the cells were trypsinized, and plated for the following experiments. Our research was approved by the Institutional Research Ethical Committee of Sun Yat-sen University (Approval number: SYSEC-KY-KS-2021-065).

\section{Chondrogenic differentiation}

As previously described ${ }^{[22-24]}$, briefly, hMSCs were trypsinized, washed, and then resuspended at a density of about $2 \times 10^{7}$ cells $/ \mathrm{mL}$ in 24 wells. They were cultured with human mesenchymal stem cell chondrogenic differentiation medium (Cyagen Biosciences Inc.). When the cells adhered, $500 \mu \mathrm{L}$ medium was added. The medium was changed every 3 days, and the induced-cartilage tissues were collected on day 7 and 14 .

\section{Transcriptome sequencing and bioinformatics analysis}

To identify the differential expression of RNA transcripts between controls and irisin-treated groups, genome-wide transcriptional sequencing was performed by Epibiotek (Guangzhou Co.,Ltd.). Differential expression analysis between controls and irisin-treated groups was performed using the DESeq2 $\mathrm{R}$ package. $P$ value was adjusted using the Hochberg and Benjamini's approach in order to control the false discovery rate. The gene expression levels were regarded to be statistically significant when the adjusted $P$ value $<0.05$ found by DESeq2. Next, Kyoto Encyclopedia of Genes and Genomes (KEGG) pathways enrichment analysis and Gene Ontology (GO) enrichment analysis were performed on the website (https://david.ncifcrf.gov). 


\section{Reverse transcription and real-time PCR}

Through the high-throughput tissue grinder, total RNAs were extracted from cartilage tissues. According to the manufacturer's protocols, reverse transcription and real-time PCR was conducted regularly, and the genes' expression levels were detected with reference to the GAPDH gene. The primer sequences were listed in Supplement Table 1.

\section{Western blot}

Cartilage tissues induced by hMSCs were ground by a high-throughput tissue grinder after adding protease inhibitor cocktail and RIPA lysis buffer. Proteins were exacted and the concentration was quantified. The proteins were subjected to $6 \%-15 \%$ SDS-PAGE gel and were then transferred to PVDF transfer membranes. The bands were blocked and incubated with primary and secondary antibodies. Finally, they were visualized with the ECL kit and quantified by the ImageJ software.

\section{Antibodies and reagents}

Antibodies against aggrecan (ACAN), collagen type II (COL2A1), and SRY-box transcription factor 9 (SOX9) were purchased from Abcam. Antibodies against PI3K/AKT signaling protein (PI3K, p-PI3K, AKT, p-AKT, mTOR, and p-mTOR), GAPDH and secondary antibodies were purchased from Cell Signaling Technology Inc.. Antibody against signal induced proliferation associated 1 like 2 (SIPA1L2) was purchased from Bioss. Human recombinant irisin was purchased from Novoprotein. 3MA (HY-19312) and GSK690693 (HY-10249) were purchased from MCE.

\section{Immunohistochemistry (IHC)}

Cartilage tissues were fixed and embedded in paraffin. The sections were deparaffinized and immersed in $65^{\circ} \mathrm{C}$ citrate buffer overnight for antigen retrieval. On the second day, the sections were incubated with $0.5 \%$ triton solution, $3 \%$ peroxidase, $5 \%$ bovine serum albumin, and anti-ACAN and anti-COL2A1 (1:100). On the next day, sections were incubated in secondary antibodies and were then detected. At last, images were obtained using an Olympus BX63 microscope.

\section{Safranin 0 and Alcian Blue staining}

After being fixed, embedded, and deparaffinized, the sections were treated with $0.5 \%$ Safranin 0 or Alcian Blue solutions. In the indicating time, sections were washed with water for 2 min. Finally, an Olympus BX63 microscope was used for photography at suitable magnifications.

\section{Immunofluorescence (IF)}

Cartilage tissue were fixed, embedded, and deparaffinized. After being treated with $0.5 \%$ triton solution and $5 \%$ bovine serum albumin, the sections were incubated with primary antibodies at the concentration of 1:100. On the second day, they were incubated with Alexa Fluor®488-labelled or Alexa Fluor ${ }^{\circledR 594-}$ conjugated secondary antibodies $(1: 100)$ for $1 \mathrm{~h}$. Next, they were labelled with DAPI and photographed with an Olympus BX63 microscope. 


\section{Plasmid transfection for SIPA1L2 over-expression}

The oligonucleotides were packaged in plasmid vectors by GeneChem (Shanghai, China). Subsequently, the OE-SIPA1L2 and OE-NC plasmid were added to the MSC-containing medium with additional Lipofectamine 3000 reagent. After $6 \mathrm{~h}$, the medium was replaced, and then the cells were cultured for following experiments.

\section{Statistical analysis}

All statistical analyses were conducted using GraphPad Software 8.0, and SPSS 20.0 statistical software package. The results were presented as mean \pm SD. Statistical analysis was performed using two-tailed independent Student's t-test or one-way ANOVA followed by Dunnett's post hoc test. A $P$ value of less than 0.05 was considered to indicate statistical significance.

\section{Results}

1. Irisin enhances chondrogenic differentiation of hMSCs.

To determine the effects of irisin on chondrogenic differentiation of hMSCs, irisin was added to chondrogenic medium at a concentration of $100 \mathrm{ng} / \mathrm{mL}$. As shown in Fig. 1A, we found that the induced cartilage tissue treated with irisin were larger than controls on day 7 and 14, respectively (Fig. 1A). Meanwhile, we detected the expression of irisin receptor integrin aV $\beta 5$ in cartilage tissues using immunofluorescence staining (Fig. 1B). Next, mRNA and proteins were extracted from induced-cartilage pellets, and results showed that irisin treatment significantly enhanced the expression levels of COL2A1, $A C A N$, and SOX9 (Fig. 1C-D). Furthermore, Safranin $\mathrm{O}$ and Alcian Blue staining were used for evaluating the metabolism of cartilage matrix, and the results revealed that irisin treatment significantly enhanced cartilage matrix synthesis in the process of chondrogenic differentiation of hMSCs both on day 7 and 14 (Fig. 1E). In addition, the results from immunohistochemistry showed that irisin treatment could upregulate the expression levels of cartilage markers (COL2A1 and ACAN) on day 7 and 14 (Fig. 1F). These outcomes showed that irisin enhanced the chondrogenic differentiation of hMSCs.

2. Irisin activates the Rap1 and PI3K/AKT pathways.

To further explore the possible mechanisms accounting for the role of irisin on the chondrogenic differentiation of hMSCs, RNA sequencing was performed in hMSC-induced cartilage tissue between controls and irisin-treated groups on day 7. As shown in Fig. 2A-B, the differentially expressed genes were screened out, and notably, irisin treatment effectively up-regulated the expression level of genes known to participate in the process of cartilage condensation and development, including COL2A1, ACAN, and COL $10 A 1$ (Fig. 2A-B). Next, GO enrichment analysis was performed and revealed that skeletal system development, collagen catabolic process, and cartilage condensation were the biological processes which were affected by irisin treatment (Fig. 2C). Furthermore, gene-set enrichment analysis (GSEA) was performed and prompted that the cartilage condensation process was up-regulated in the irisin-treated 
group (ES=0.746, NES=1,685, and $P<0.05$ ), and the relative genes (COL2A1, ACAN, ROR2, MGP, RUNX2, and COL 11A1) were significantly up-regulated (Fig. 2D). Besides, KEGG pathway analysis identified that the Rap1 and PI3K/AKT signaling pathway were activated in the irisin-treated group (Fig. 2E). Verification of the special gene expressions in Rap1 signaling pathway was performed, and we found that the expression of SIPA1L2 was substantially suppressed in the irisin-treated group (Fig. 2F).

3. Irisin activates the Rap1 signaling pathway by suppressing the expression of SIPA1L2.

To investigate the function of Rap1 signaling pathway in the process of chondrogenic differentiation, we subsequently assessed the expression level of the key factor, SIPA1L2, a Rap1 GTPase activating protein, which was significantly inhibited with irisin treatment (Fig. 2A \& 2F). The results from Western blot and immunofluorescence showed that the expression level of SIPA1L2 was evidently down-regulated with irisin treatment (Fig. 3A-B). To explore the capability of SIPA1L2 in the process of chondrogenic differentiation, SIPA1L2 was over-expressed using plasmid transfection, and the over-expression efficiency was tested using Western blot (Fig. 3C). As shown in Supplement Fig. 1, we found that the induced-cartilage pellets treated with irisin were larger than controls, while this change was obviously inhibited in the OE-SIPA1L2 group (Supplement Fig. 1). Subsequently, we collected the hMSCs-induced cartilage tissue on day 7 , and the results from $\mathrm{PPCR}$, Western blot and immunofluorescence revealed that OE-SIPA1L2 significantly inhibited the expression of COL2A1, ACAN, and SOX9, while activated the $\mathrm{PI} 3 \mathrm{~K} / \mathrm{AKT} / \mathrm{mTOR}$ pathway (Fig. 3D-F ). The results from Alcian Blue and Safranin 0 staining also showed that OE-SIPA1L2 could negatively regulate cartilage matrix synthesis in the irisin-treated group (Fig. 3G). These findings indicated that the expression level of SIPA1L2 was suppressed, and the Rap1 signaling pathway was activated with irisin treatment.

4. Irisin enhances chondrogenic differentiation of hMSCs by activating the PI3K/AKT pathway.

It has been demonstrated by previous studies, the PI3K/AKT signaling pathway, which was involved in the process of chondrogenic differentiation, was regulated by Rap1 signaling pathway ${ }^{[25-26]}$. Meanwhile, in our study the activation of the PI3K/AKT signaling pathway was detected in irisin-treated group by KEGG analysis (Fig. 2E). Hence, the expression level of the key molecules of the PI3K/AKT pathway were detected. As shown in Fig. 4A-C, the phosphorylation levels of PI3K and AKT as well as the ratio of $p$ $\mathrm{PI3K} / \mathrm{PI}$ IKK and p-AKT/AKT were positively regulated with irisin treatment (Fig. 4A-C). To further explore the mechanism, two well-established small-molecule antagonists of PI3K/AKT pathway, 3-Methyladenine (3MA, a PI3K inhibitor) and GSK690693 (an ATP-competitive pan-AKT inhibitor) were employed.

Compared with the control group, irisin treatment obviously enlarged the cartilage pellets, while 3MA and GSK690693 treatment decreased the diameter of cartilage tissues (Supplement Fig. 2). In addition, the detection of cartilage matrix components (COL2A1, ACAN, and SOX9) revealed that irisin promoted chondrogenic differentiation, while 3MA and GSK690693 inhibited these changes (Fig. 4D-E). And the results from immunofluorescence were consistent with the abovementioned findings (Fig. 4F). Next, to evaluate the cartilage matrix synthesis and accumulation, Safranin $\mathrm{O}$ and Alcian Blue staining were used. Our data suggested that irisin treatment could promote the synthesis of cartilage matrix, while the effects 
were partially reversed by 3MA and GSK690693 (Fig. 4G). These results demonstrated that the PI3K/AKT pathway was activated on the process of chondrogenic differentiation of irisin-induced hMSCs.

\section{Irisin mediated up-regulation of miR-125b-5p targeting SIPA1L2 to promote chondrogenic differentiation of hMSCs.}

It has been found that miRNAs were involved in regulating the chondrogenic differentiation of hMSCs ${ }^{[22,}$

27]. We compared miRNA expression in controls and irisin-treated cartilage tissue (Fig. 5A), and we selected 12 genes among these differentially over-expressed miRNAs-targeting SIPA1L2 gene from the database, and then we detected the expression levels of these miRNAs, and the results from qPCR showed that miR-125b-5p was most significantly up-regulated with irisin treatment (Fig. 5B). Thus, we transduced hMSCs with miR-125b-5p mimics and irisin-treated hMSCs with miR-125b-5p inhibitor. As expected, the results showed that the expression levels of chondrogenic markers (COL2A1, ACAN, and SOX9) were up-regulated with miR-125b-5p mimics transduction, while being down-regulated in irisintreated hMSCs with miR-125b-5p inhibitor transduction (Fig. 5C-D). Consistently, the results from immunofluorescence showed that miR-125b-5p mimics inhibited the level of SIPA1L2 and increased the expressions of typical chondrogenic markers COL2A1 and SOX9. Correspondingly, the transduction of hMSCs with miR-125b-5p inhibitor partly reversed the expression level of SIPA1L2 under irisin treatment and abrogated the positive effects of irisin on COL2A1 and SOX9 expression levels (Fig. 5E-F). Moreover, Alcian Blue and Safranin $O$ staining showed more abundant cartilage matrix synthesis in the $m i R-125 b$ $5 p$ transduction group compared with the controls (Fig. $5 \mathrm{G}$ ). On the contrary, the promotional effect of irisin on chondrogenic differentiation was abrogated by transducting miR-125b-5p inhibitor (Fig. $5 \mathrm{H}$ ). These data suggested that irisin treatment could up-regulate miR-125b-5p targeting SIPA1L2, enhancing the chondrogenic differentiation of hMSCs.

\section{Discussion}

Irisin, known as a polypeptide fragment of FNDC5, produced in abundance by skeletal muscle, is a hormone-like myokine that leads to increased energy expenditure as well as regulation of glucose homeostasis and bone metabolism ${ }^{[11-12,28]}$. Ample evidence is emerging in support of irisin's physiological relevance in development of the skeletal system and chondroprotective actions ${ }^{[20-21,28]}$. Moreover, with a wide spectrum for the repair of defective or damaged cartilage tissue, MSCs are currently undergoing trials for articular cartilage repair in the field of tissue engineering ${ }^{\text {[29-32] }}$. Nevertheless, the effects and potential mechanisms of irisin on chondrogenic differentiation of hMSCs are elusive yet.

In our study, we found that irisin played a promoting role on chondrogenic differentiation of hMSCs. Mechanism-wise, we demonstrated that irisin activated the Rap1 signaling pathway and PI3K/AKT pathway. Further research revealed that irisin increased the level of miR-125b-5p, targeting SIPA1L2, which regulated the Rap1/PI3K/AKT axis as a Rap1 GTPase activating proteins and finally promoted the expressions of chondrogenic differentiation genes COL2A1, ACAN, and SOX9 (Fig. 6). Our research 
evidenced the positive effects of irisin on chondrogenic differentiation of hMSCs, and detected its molecular mechanism that may lead to new ideas for the application of hMSCs implantation in the field of tissue engineering to repair articular cartilage lesions.

Rap1 protein, as a member of the Ras superfamily of small GTPases, is regulated by both Rap1 GTPase activating proteins and Rap1-specific guanine nucleotide exchange factors, which can activate the following ERK signaling, PI3K/AKT signaling and other downstream pathways ${ }^{[33-35]}$. As a Rap1 GTPase activating protein, SIPA1L2 promotes the intrinsic GTPase activity of Rap1 that catalyzes the hydrolysis of GTP to GDP, and consequently, inhibits the downstream pathways including ERK signaling and $\mathrm{PI} 3 \mathrm{~K} / \mathrm{AKT}$ signaling ${ }^{[36-37]}$. According to available evidence, the PI3K/AKT signaling pathway was essential for normal cartilage development, metabolism, and degradation $[25-26,38-39]$. Nevertheless, the molecular mechanisms governing Rap1/PI3K/AKT on the regulation of chondrogenic differentiation are still unclear. Our results suggested that irisin treatment effectively down-regulated the expression of SIPA1L2, helped to maintain activation of long-term Rap1 protein, and activated the downstream $\mathrm{PISK} / \mathrm{AKT}$ pathway in the process of induced-chondrogenic differentiation of hMSCs.

MiRNAs are short, noncoding, endogenous oligonucleotides which can be found in almost all cells. Through post-transcriptional gene silencing, they can regulate gene expression $[27,40]$. Accumulated evidences have shown that post-transcriptional regulation by microRNAs (miRNAs) was involved in the osteogenic and chondrogenic differentiation of MSCs ${ }^{[27,41]}$. It was a practical approach to mediate the chondrogenic differentiation of MSCs by manipulating the expression of specific miRNAs ${ }^{[42-44]}$. A variety of miRNAs participated in the processes of irisin on osteogenic differentiation of MSCs, browning of white adipocytes, and anti-inflammation ${ }^{[19,45-46]}$. Nevertheless, whether miRNAs participate in the process of irisin-enhanced chondrogenic differentiation of hMSCs is still unknown. Our study illustrated that irisin elevated chondrogenic differentiation in hMSCs through up-regulation of miR-125b-5p targeting SIPA1L2. Correspondingly, the administration of miR-125b-5p mimics successfully enhanced the chondrogenic differentiation. Meanwhile, the transduction of $m i R-125 b-5 p$ inhibitor practically abrogated the regulative effects of irisin on hMSCs' chondrogenic differentiation. Thus, we hypothesize that it could be an effective therapy to modify hMSCs with miRNAs for diseases involving cartilage injury in the future.

\section{Conclusions}

Collectively, we described that irisin effectively enhanced chondrogenic differentiation of hMSCs. Mechanistically, it was identified that irisin may activate the Rap1/PI3K/AKT signaling axis by upregulating miR-125b-5p targeting SIPA1L2. In addition, the transductions of miR-125b-5p mimics or inhibitors can simulate or reverse the effects respectively. More studies are warranted in the future to explore the application of irisin on tissue engineering and the clinical translation on cartilage regeneration.

\section{Abbreviations}




\begin{tabular}{|ll|}
\hline Abbreviations & Descriptions \\
\hline hMSCs & human mesenchymal stem cells \\
FNDC5 & fibronectin component including protein 5 \\
ACAN & aggrecan \\
COL2A1 & collagen type II \\
SOX9 & SRY-box transcription factor 9 \\
SIPA1L2 & signal induced proliferation associated 1 like 2 \\
\hline
\end{tabular}

\section{Declarations}

Acknowledgements

Not applicable.

\section{Authors' contributions}

WJG and DSH designed the experiments. TQC, YP, WJH, HSS, PFL, YCQ, JCQ, XJQ, BG, HZ, YBC, YXZ, SGL, AJL, WJG, and DSH conducted the experiments. TQC, YP, and WJH acquired the data. TQC, WJG, and DSH analyzed the data. TQC, YP, WJH, WJG, and DSH wrote the manuscript. All authors read and approved the final manuscript.

\section{Funding}

This work was supported by the grants from the National Natural Science Foundation of China (No. 81572134); Guangdong Basic and Applied Basic Research Foundation (No. 2019A1515110122 \& No. 2021A1515012619); Fundamental Research Funds of the Central Universities (No. 20ykpy94); Science and Technology Program of Guangzhou, China (No. 202102010159); and Sun Yat-sen Scholarship for Young Scientist for Wenjie Gao.

\section{Data Availability}

The data used to support the findings of this study are available from the corresponding authors upon request.

\section{Ethics approval and consent to participate}

Our research was approved by the Institutional Research Ethical Committee of Sun Yat-sen University (Approval number: SYSEC-KY-KS-2021-065).

\section{Conflicts of Interest}


We declare that there is no conflict of interest regarding the publication of this paper.

\section{Author details}

${ }^{1}$ Department of Orthopedics, Sun Yat-sen Memorial Hospital of Sun Yat-sen University, \#107 West Yan Jiang Road, Guangzhou, Guangdong, China.

\section{References}

1. Cancedda R, Dozin B, Giannoni P, Quarto R. Tissue engineering and cell therapy of cartilage and bone. Matrix Biol. 2003 Mar;22(1):81-91.

2. Li T, Liu B, Chen K, Lou Y, Jiang Y, Zhang D. Small molecule compounds promote the proliferation of chondrocytes and chondrogenic differentiation of stem cells in cartilage tissue engineering. Biomed Pharmacother. 2020 Nov; 131:110652.

3. Dieppe PA, Lohmander LS. Pathogenesis and management of pain in osteoarthritis. Lancet. 2005 Mar;12-18(9463):965-73. 365(.

4. Griffın MF, Ibrahim A, Seifalian AM, Butler PEM, Kalaskar DM, Ferretti P. Argon plasma modification promotes adipose derived stem cells osteogenic and chondrogenic differentiation on nanocomposite polyurethane scaffolds; implications for skeletal tissue engineering. Mater Sci Eng C Mater Biol Appl. 2019 Dec;105:110085.

5. Mistry AS, Mikos AG. Tissue engineering strategies for bone regeneration. Adv Biochem Eng Biotechnol. 2005;94:1-22.

6. Battafarano G, Rossi M, De Martino V, Marampon F, Borro L, Secinaro A, Del Fattore A. Strategies for Bone Regeneration: From Graft to Tissue Engineering. Int J Mol Sci. 2021 Jan 23;22(3):1128.

7. Wescoe KE, Schugar RC, Chu CR, Deasy BM. The role of the biochemical and biophysical environment in chondrogenic stem cell differentiation assays and cartilage tissue engineering. Cell Biochem Biophys. 2008;52(2):85-102.

8. Kim YG, Choi J, Kim K. Mesenchymal Stem Cell-Derived Exosomes for Effective Cartilage Tissue Repair and Treatment of Osteoarthritis. Biotechnol J. 2020 Dec;15(12):e2000082.

9. Huh JY, Mougios V, Skraparlis A, Kabasakalis A, Mantzoros CS. Irisin in response to acute and chronic whole-body vibration exercise in humans. Metabolism. 2014 Jul;63(7):918-21.

10. Boström P, Wu J, Jedrychowski MP, Korde A, Ye L, Lo JC, Rasbach KA, Boström EA, Choi JH, Long JZ, Kajimura S, Zingaretti MC, Vind BF, Tu H, Cinti S, Højlund K, Gygi SP, Spiegelman BM. A PGC1-adependent myokine that drives brown-fat-like development of white fat and thermogenesis. Nature. 2012 Jan 11;481(7382):463-8.

11. Colaianni G, Cinti S, Colucci S, Grano M. Irisin and musculoskeletal health. Ann N Y Acad Sci. 2017 Aug;1402(1):5-9.

12. Perakakis N, Triantafyllou GA, Fernández-Real JM, Huh JY, Park KH, Seufert J, Mantzoros CS. Physiology and role of irisin in glucose homeostasis. Nat Rev Endocrinol. 2017 Jun;13(6):324-37. 
13. Lee P, Linderman JD, Smith S, Brychta RJ, Wang J, Idelson C, Perron RM, Werner CD, Phan GQ, Kammula US, Kebebew E, Pacak K, Chen KY, Celi FS. Irisin and FGF21 are cold-induced endocrine activators of brown fat function in humans. Cell Metab. 2014 Feb 4;19(2):302-9.

14. Park EJ, Myint PK, Ito A, Appiah MG, Darkwah S, Kawamoto E, Shimaoka M. Integrin-Ligand Interactions in Inflammation, Cancer, and Metabolic Disease: Insights Into the Multifaceted Roles of an Emerging Ligand Irisin. Front Cell Dev Biol. 2020 Oct;26:8:588066.

15. Zhang D, Tan X, Tang N, Huang F, Chen Z, Shi G. Review of Research on the Role of Irisin in Tumors. Onco Targets Ther. 2020 May;19:13:4423-30.

16. Colaianni G, Mongelli T, Cuscito C, Pignataro P, Lippo L, Spiro G, Notarnicola A, Severi I, Passeri G, Mori G, Brunetti G, Moretti B, Tarantino U, Colucci SC, Reseland JE, Vettor R, Cinti S, Grano M. Irisin prevents and restores bone loss and muscle atrophy in hind-limb suspended mice. Sci Rep. 2017 Jun;6(1):2811. 7(.

17. Metzger CE, Anand Narayanan S, Phan PH, Bloomfield SA. Hindlimb unloading causes regional loading-dependent changes in osteocyte inflammatory cytokines that are modulated by exogenous irisin treatment. NPJ Microgravity. 2020 Oct; 7:6:28.

18. Posa F, Colaianni G, Di Cosola M, Dicarlo M, Gaccione F, Colucci S, Grano M, Mori G. The Myokine Irisin Promotes Osteogenic Differentiation of Dental Bud-Derived MSCs. Biology (Basel). $2021 \mathrm{Apr}$ 3;10(4):295.

19. Liu C, Liu AS, Zhong D, Wang CG, Yu M, Zhang HW, Xiao H, Liu JH, Zhang J, Yin K. Circular. RNA AFF4 modulates osteogenic differentiation in BM-MSCs by activating SMAD1/5 pathway through miR-135a-5p/FNDC5/Irisin axis. Cell Death Dis. 2021 Jun 18;12(7):631.

20. Li X, Zhu X, Wu H, Van Dyke TE, Xu X, Morgan EF, Fu W, Liu C, Tu Q, Huang D, Chen J. Roles and Mechanisms of Irisin in Attenuating Pathological Features of Osteoarthritis. Front Cell Dev Biol. 2021 Sep;28:9:703670.

21. Wang FS, Kuo CW, Ko JY, Chen YS, Wang SY, Ke HJ, Kuo PC, Lee CH, Wu JC, Lu WB, Tai MH, Jahr H, Lian WS. Irisin Mitigates Oxidative Stress, Chondrocyte Dysfunction and Osteoarthritis Development through Regulating Mitochondrial Integrity and Autophagy. Antioxidants (Basel). 2020 Sep $1 ; 9(9): 810$.

22. Wu Z, Qiu X, Gao B, Lian C, Peng Y, Liang A, Xu C, Gao W, Zhang L, Su P, Rong L, Huang D. Melatoninmediated miR-526b-3p and miR-590-5p upregulation promotes chondrogenic differentiation of human mesenchymal stem cells. J Pineal Res. 2018 Aug;65(1):e12483.

23. Gao W, Lin M, Liang A, Zhang L, Chen C, Liang G, Xu C, Peng Y, Chen C, Huang D, Su P. Melatonin enhances chondrogenic differentiation of human mesenchymal stem cells. J Pineal Res. 2014 Jan;56(1):62-70.

24. Gao B, Gao W, Wu Z, Zhou T, Qiu X, Wang X, Lian C, Peng Y, Liang A, Qiu J, Zhu Y, Xu C, Li Y, Su P, Huang D. Melatonin rescued interleukin $1 \beta$-impaired chondrogenesis of human mesenchymal stem cells. Stem Cell Res Ther. 2018 Jun 14;9(1):162. 
25. Sun K, Luo J, Guo J, Yao X, Jing X, Guo F. The PI3K/AKT/mTOR signaling pathway in osteoarthritis: a narrative review. Osteoarthritis Cartilage. 2020 Apr;28(4):400-9.

26. Fujita T, Azuma Y, Fukuyama R, Hattori Y, Yoshida C, Koida M, Ogita K, Komori T. Runx2 induces osteoblast and chondrocyte differentiation and enhances their migration by coupling with PI3K-Akt signaling. J Cell Biol. 2004 Jul;5(1):85-95. 166(.

27. Iaquinta MR, Lanzillotti C, Mazziotta C, Bononi I, Frontini F, Mazzoni E, Oton-Gonzalez L, Rotondo JC, Torreggiani E, Tognon M, Martini F. The role of microRNAs in the osteogenic and chondrogenic differentiation of mesenchymal stem cells and bone pathologies. Theranostics. $2021 \mathrm{Apr}$ 30;11(13):6573-6591.

28. Colaianni G, Sanesi L, Storlino G, Brunetti G, Colucci S, Grano M. Irisin and Bone: From Preclinical Studies to the Evaluation of Its Circulating Levels in Different Populations of Human Subjects. Cells. 2019 May 14;8(5):451.

29. Armiento AR, Stoddart MJ, Alini M, Eglin D. Biomaterials for articular cartilage tissue engineering: Learning from biology. Acta Biomater. 2018 Jan;65:1-20.

30. Gupta PK, Das AK, Chullikana A, Majumdar AS. Mesenchymal stem cells for cartilage repair in osteoarthritis. Stem Cell Res Ther. 2012 Jul;9(4):25. 3(.

31. Li J, Pei M. Cell senescence: a challenge in cartilage engineering and regeneration. Tissue Eng Part $B$ Rev. 2012 Aug;18(4):270-87.

32. Roseti L, Desando G, Cavallo C, Petretta M, Grigolo B. Articular Cartilage Regeneration in Osteoarthritis. Cells. 2019 Oct;23(11):1305. 8(.

33. Boettner B, Van Aelst L. Control of cell adhesion dynamics by Rap1 signaling. Curr Opin Cell Biol. 2009 Oct;21(5):684-93.

34. Gold MR, Ingham RJ, McLeod SJ, Christian SL, Scheid MP, Duronio V, Santos L, Matsuuchi L. Targets of B-cell antigen receptor signaling: the phosphatidylinositol 3-kinase/Akt/glycogen synthase kinase3 signaling pathway and the Rap1 GTPase. Immunol Rev. 2000 Aug;176:47-68.

35. Shah S, Brock EJ, Ji K, Mattingly RR. Ras and Rap1: A tale of two GTPases. Semin Cancer Biol. 2019 Feb;54:29-39.

36. Andres-Alonso M, Ammar MR, Butnaru I, Gomes GM, Acuña Sanhueza G, Raman R, Yuanxiang P, Borgmeyer M, Lopez-Rojas J, Raza SA, Brice N, Hausrat TJ, Macharadze T, Diaz-Gonzalez S, Carlton M, Failla AV, Stork O, Schweizer M, Gundelfinger ED, Kneussel M, Spilker C, Karpova A, Kreutz MR. SIPA1L2 controls trafficking and local signaling of TrkB-containing amphisomes at presynaptic terminals. Nat Commun. 2019 Nov 29;10(1):5448.

37. Tao F, Beecham GW, Rebelo AP, Svaren J, Blanton SH, Moran JJ, Lopez-Anido C, Morrow JM, Abreu L, Rizzo D, Kirk CA, Wu X, Feely S, Verhamme C, Saporta MA, Herrmann DN, Day JW, Sumner CJ, Lloyd TE, Li J, Yum SW, Taroni F, Baas F, Choi BO, Pareyson D, Scherer SS, Reilly MM, Shy ME, Züchner S, Inherited Neuropathy Consortium. Variation in SIPA1L2 is correlated with phenotype modification in Charcot- Marie- Tooth disease type 1A. Ann Neurol. 2019 Mar;85(3):316-30. 
38. Chen L, Qanie D, Jafari A, Taipaleenmaki H, Jensen CH, Säämänen AM, Sanz ML, Laborda J, Abdallah BM, Kassem M. Delta-like 1/fetal antigen-1 (Dlk1/FA1) is a novel regulator of chondrogenic cell differentiation via inhibition of the Akt kinase-dependent pathway. J Biol Chem. 2011 Sep 16;286(37):32140-9.

39. McMahon LA, Prendergast PJ, Campbell VA. A comparison of the involvement of $p 38, E R K 1 / 2$ and $\mathrm{PI} 3 \mathrm{~K}$ in growth factor-induced chondrogenic differentiation of mesenchymal stem cells. Biochem Biophys Res Commun. 2008 Apr 18;368(4):990-5.

40. Lu TX, Rothenberg ME. MicroRNA. J Allergy Clin Immunol. 2018 Apr;141(4):1202-7.

41. Wang X, Chen T, Deng Z, Gao W, Liang T, Qiu X, Gao B, Wu Z, Qiu J, Zhu Y, Chen Y, Liang Z, Zhou H, Xu C, Liang A, Su P, Peng Y, Huang D. Melatonin promotes bone marrow mesenchymal stem cell osteogenic differentiation and prevents osteoporosis development through modulating circ_0003865 that sponges miR-3653-3p. Stem Cell Res Ther. 2021 Feb;25(1):150. 12.

42. Kenyon JD, Sergeeva O, Somoza RA, Li M, Caplan Al, Khalil AM, Lee Z. Analysis of $-5 p$ and $-3 p$ Strands of miR-145 and miR-140 During Mesenchymal Stem Cell Chondrogenic Differentiation. Tissue Eng Part A. 2019 Jan;25(1-2):80-90.

43. Xu S, Wu X. miR-134 inhibits chondrogenic differentiation of bone marrow mesenchymal stem cells by targetting SMAD6. Biosci Rep. 2019 Jan 30;39(1): BSR20180921.

44. Won Lee G, Thangavelu M, Joung Choi M, Yeong Shin E, Sol Kim H, Seon Baek J, Woon Jeong Y, Eun Song J, Carlomagno C, Miguel Oliveira J, Luis Reis R, Khang G. Exosome mediated transfer of miRNA-140 promotes enhanced chondrogenic differentiation of bone marrow stem cells for enhanced cartilage repair and regeneration. J Cell Biochem. 2020 Jul;121(7):3642-52.

45. Ge X, Sathiakumar D, Lua BJ, Kukreti H, Lee M, McFarlane C. Myostatin signals through miR-34a to regulate Fndc5 expression and browning of white adipocytes. Int J Obes (Lond). 2017 Jan;41(1):137-48.

46. Ma LY, Liu JM, Du GL, Dang XB. Irisin attenuates lipopolysaccharide-induced acute lung injury by downregulating inflammatory cytokine expression through miR-199a-mediated Rad23b overexpression. Exp Cell Res. 2021 Jul 15;404(2):112593.

\section{Figures}


A

B
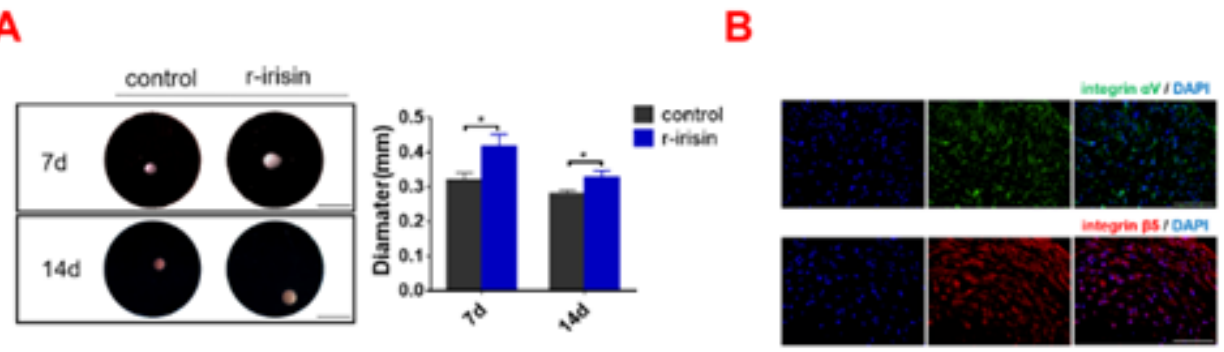

C

D
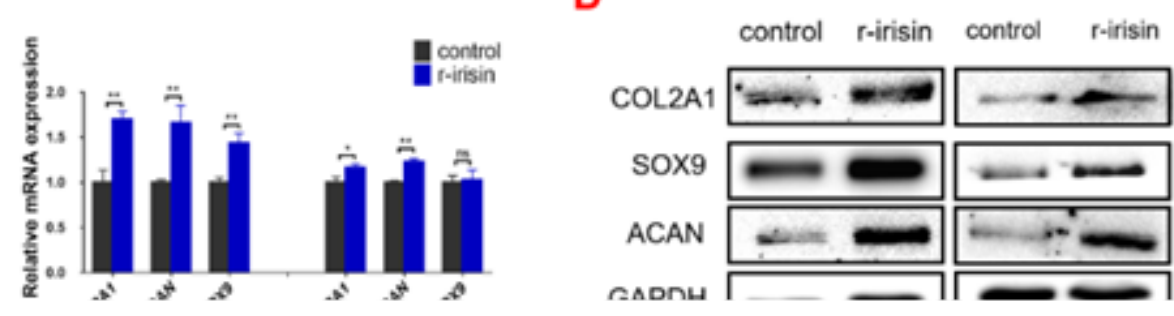

\section{Figure 1}

Irisin enhances chondrogenic differentiation of hMSCs. (A) Macro-images of induced-cartilage tissues were taken on Day-7 and Day-14 (Left panel). (Magnification: $\times 15$, scale bar: $2 \mathrm{~mm}$ ). The relative diameter of cartilage tissues in controls and r-irisin-treated group (Right panel). (B) The expressions of integrin aV 35 receptor in hMSCs-induced cartilage tissue. (Magnification: $\times 400$, scale bar: $50 \mu \mathrm{m}$ ). (C) mRNA expression of COL2A1, ACAN and SOX9 were detected by qPCR in different groups. (D) Protein expression of COL2A1, ACAN and SOX9 were detected by western blot in different groups. (E) Safranin 0 and Alcian Blue staining for cartilage matrix metabolism on 7 and 14 days in different groups. (Magnification: $\times 200$, scale bar: $100 \mu \mathrm{m})(\mathrm{F})$ Immunohistochemistry for COL2A1 and ACAN on 7 and 14 days in different groups. (Magnification: $\times 200$, scale bar: $100 \mu \mathrm{m}$ ) The levels were determined using the Image J software. 
The concentration of irisin used in this figure was $100 \mathrm{ng} / \mathrm{ml} .{ }^{*} P<0.05,{ }^{* *} P<0.01$ compared with the control-group.

\section{Figure 2}

Irisin activates the Rap1 and PI3K/AKT pathways. (A) The heatmap of DEGs, as detected by RNA-seq in hMSCs induced-cartilage tissue treated with and without irisin for $7 \mathrm{~d}(\mathrm{n}=3$ biological replicates). (B) The volcano map of genes distribution. (C) GO terms with the most significant $\mathrm{P}$ values. BP: biological process, CC: cellular component, MF: molecular function. (D) GSEA of positively associated cartilage condensation process (Left panel) and cartilage condensation hallmark genes treated with irisin compared with vehicle (Right panel). (E) The top 20 of pathways enrichment by KEGG analysis. (F) The mRNA expression of key genes (RAP1A, RAP1B, RAP1GAP, SIPA1L2, RASGRP) in Rap1 signaling pathway. The concentration of irisin used in this figure was $100 \mathrm{ng} / \mathrm{ml} .{ }^{*} P<0.05,{ }^{* \star} P<0.01$ compared to control.

\section{Figure 3}

Irisin activates the Rap1 signaling pathway by suppressing the expression of SIPA1L2. (A) Protein expression level of SIPA1L2 were detected by western blot in control and irisin-induced group. (B) Immunohistochemistry for SIPA1L2 on 7 and 14 days in different groups. (Magnification: $\times 400$, scale bar: $50 \mu \mathrm{m})$. (C) Transfection efficiency verification of SIAP1L2 by western blot. (D) mRNA expressions of COL2A1, ACAN, and SOX9. (E) Immunofluorescence of COL2A1 and SOX9 of MSCs-induced cartilage tissue on 7 days. (Magnification: $\times 400$, scale bar: $50 \mu \mathrm{m}$ ). (F) Protein expression of PI3K, p-PI3K, AKT, pAKT, mTOR, p-mTOR, COL2A1, ACAN and SOX9 were detected by western blot in different groups. (G) Safranin $O$ and Alcian blue staining in different groups. (Magnification: $\times 200$, scale bar: $100 \mu \mathrm{m})$. The concentration of irisin used in this figure was $100 \mathrm{ng} / \mathrm{ml} .{ }^{*} P<0.05,{ }^{* \star} P<0.01$ compared with the control group. ${ }^{\#} P<0.05,{ }^{\# \#} P<0.01$ compared with the irisin + OE-NC group.

\section{Figure 4}

Irisin enhances chondrogenic differentiation of hMSCs by activating the PI3KVAKT pathway. (A) Protein expression levels of PI3K, p-PI3K, AKT and p-AKT were detected by western blot in controls and irisintreated groups on 7 days. (B) Quantitative expressions levels of PI3K, p-PI3K, AKT and p-AKT using Image $\mathrm{J}$ software. (C) The ratios of relative protein expression of $\mathrm{p}-\mathrm{PI} 3 \mathrm{~K}$ to relative protein expression of $\mathrm{PI} 3 \mathrm{~K}(\mathrm{p}$ $\mathrm{PI} 3 \mathrm{~K} / \mathrm{PI} 3 \mathrm{~K})$, and $\mathrm{p}-\mathrm{AKT}$ to relative protein expression of AKT (p-AKT/AKT). (D) The mRNA and protein expressions of COL2A1, ACAN, and SOX9 were detected by qPCR and western blot in control, r-irisin, and 
r-irisin+3MA-treated group. (E) The mRNA and protein expressions of COL2A1, ACAN, and SOX9 were detected by qPCR and western blot in control, irisin, and irisin+GSK690693-treated group. (F) Immunofluorescence of COL2A1 and SOX9 of hMSCs-induced cartilage tissues. (Magnification: $\times 400$, scale bar: $50 \mu \mathrm{m}$ ). (G) Safranin $\mathrm{O}$ and Alcian Blue staining were performed after 7 days of differentiation. (Magnification: $\times 200$, scale bar: $100 \mu \mathrm{m}$ ). The concentration of irisin used in this figure was $100 \mathrm{ng} / \mathrm{ml} .{ }^{*} P$ $<0.05,{ }^{* \star} P<0.01$ compared with the control group. ${ }^{\#} P<0.05,{ }^{\# \#} P<0.01$ compared with the irisin-treated group.

A

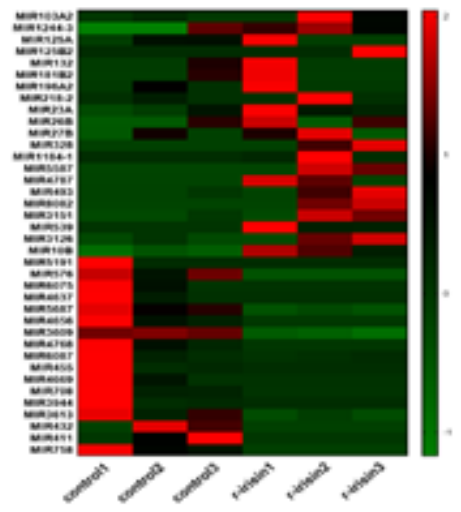

C

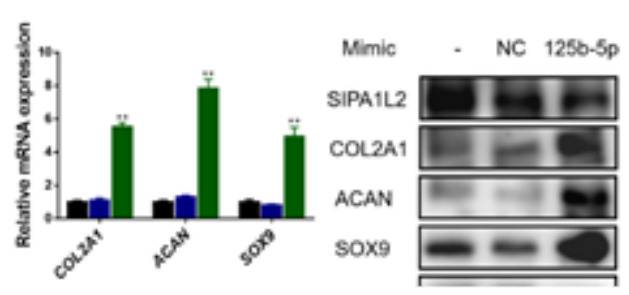

B

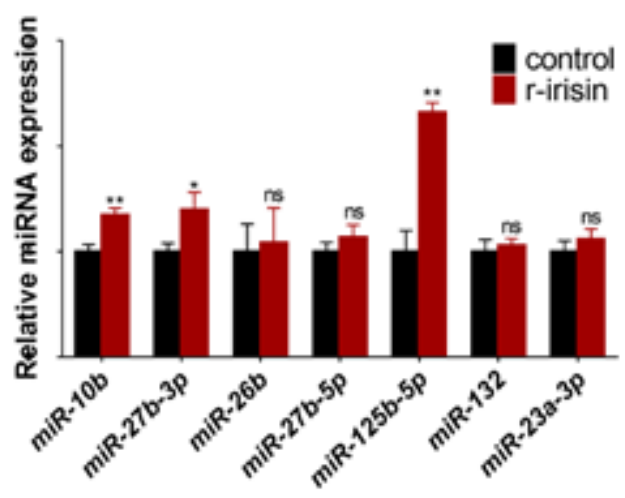

D

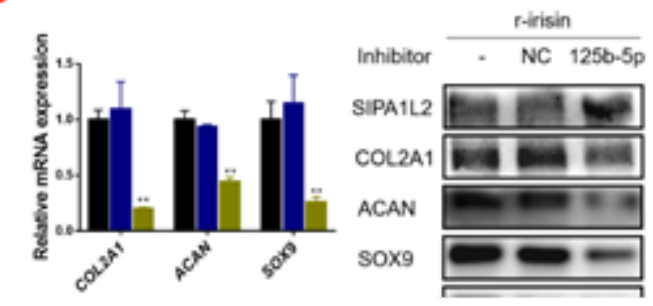


Irisin mediated up-regulation of miR-125b-5p targeting SIPA1L2 to promote chondrogenic differentiation of hMSCs. (A) Human MSCs was seeded in 24-wells plates via high-density micromass culture, and chondrogenic differentiation was induced in chondrogenic medium with or without irisin $(100 \mathrm{ng} / \mathrm{ml})$ treatment for 7 days. Differently miRNA expressions in control and irisin-treated group were shown. (B) Relative expressions of the indicated miRNAs in cartilage tissues detected by qPCR. (C) The mRNA expressions (Left panel) of COL2A1, ACAN, and SOX9, and the protein expressions (Right panel) of SIPA1L2, COL2A1, ACAN, and SOX9 in the control, $N C$, and miR-125b-5p mimics transduction groups. (D) The mRNA expressions (Left panel) of COL2A1, ACAN, and SOX9, and the protein expressions (Right panel) of SIPA1L2, COL2A1, ACAN, and SOX9 in the control, $N C$, and miR-125b-5p inhibitor transduction groups under irisin treatment. (E-F) The expressions of SIPA1L2, COL2A1, and SOX9 were detected by immunofluorescence combined with DAPI staining for the nuclei in different groups. (Magnification: $\times 400$, scale bar: $50 \mu \mathrm{m})$. (G-H) Safranin 0 and Alcian Blue staining were performed in cartilage tissue paraffin sections with different treatments as shown. (Magnification: $\times 200$, scale bar: $100 \mu \mathrm{m})$. The concentration of irisin used in this figure was $100 \mathrm{ng} / \mathrm{ml} .{ }^{*} P<0.05,{ }^{* *} P<0.01$ compared with the control group or NC group.

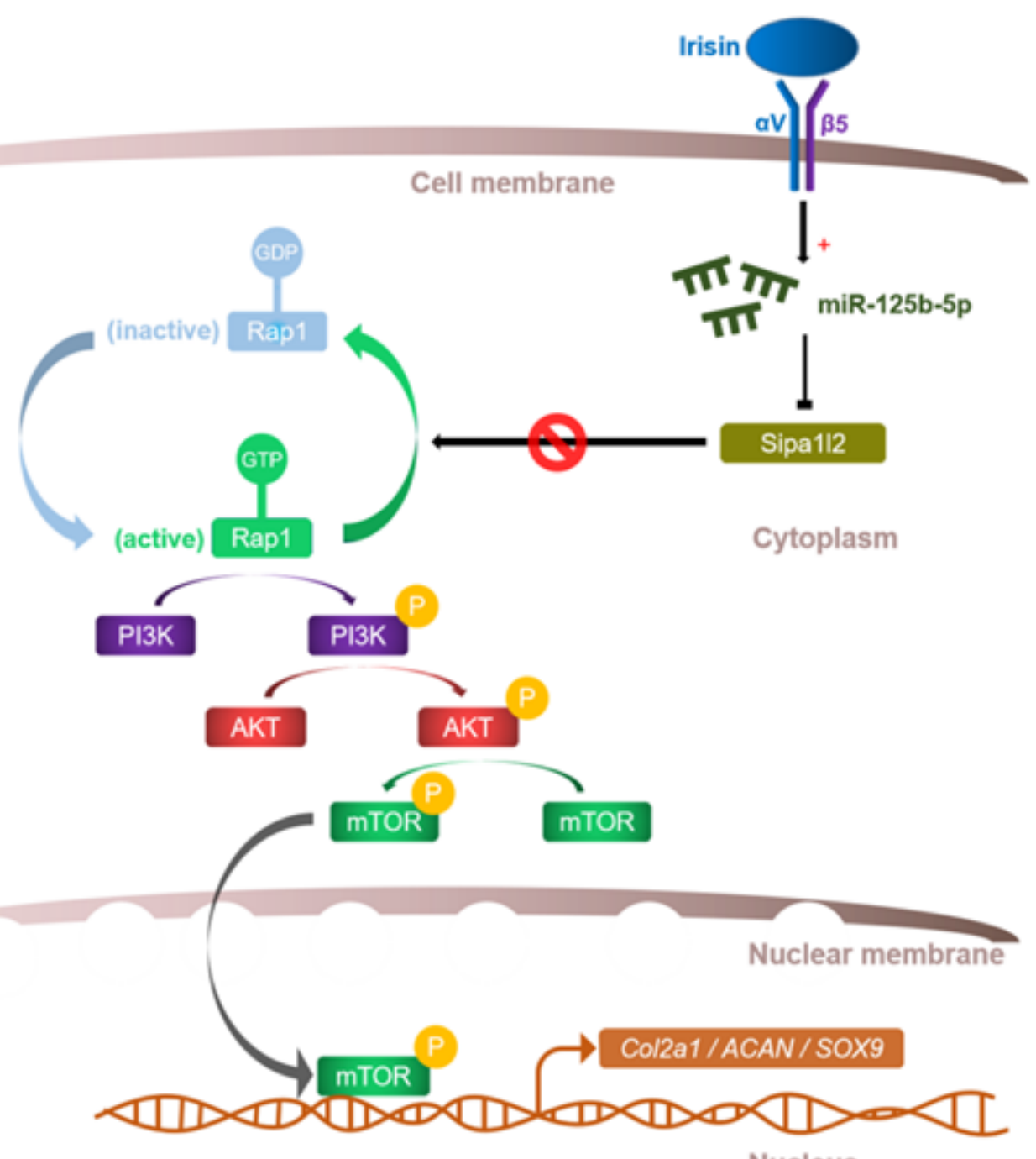

Nucleus 
Figure 6

Schematic diagram highlighting the primary findings of this study. Irisin up-regulates miR-125b-5p targeting SIPA1L2 and activates the Rap1/PI3K/AKT signaling axis, and consequently promoted the expressions of chondrogenic differentiation genes (COL2A1, ACAN, and SOX9), enhancing chondrogenic differentiation in hMSCs via integrin repectors.

\section{Supplementary Files}

This is a list of supplementary files associated with this preprint. Click to download.

- MSCsSupplementTableandFigures.docx 